\title{
Fatal Sepsis and Multiorgan Failure Following Bacillus Calmette-Guerin Instillation for Bladder Cancer Treatment
}

\section{Carlo Moreschi ${ }^{1}$ and Ugo Da Broi ${ }^{2 *}$}

${ }^{1}$ Department of Medical and Biological Sciences, Section of Forensic Medicine, University of Udine, Italy

${ }^{2}$ Department of Medical and Biological Sciences, Section of Forensic Medicine, University of Udine, Italy

\begin{abstract}
A Fatal sepsis and multiple organ failure occurred in a 61-year-old man, seven days after the anti-tumoral instillation of bacillus Calmette-Guérin for bladder carcinoma treatment. Autopsy findings revealed uncommon diffuse miliary caseating granulomas involving lungs. Judicial Authority censured the urology surgical team for delayed diagnosis and incorrect therapeutical intervention, not applied when early local and systemic adverse effects arose, in absence of exhaustive information given to the patient on all post-operative complications.
\end{abstract}

Keywords: Bladder cancer; BCG instillation; Sepsis; Multiorgan failure

\section{Introduction}

Local bacillus Calmette-Guérin (BCG) immunotherapy is an effective and widely used treatment for superficial bladder carcinoma but its application in clinical practice remains controversial due to several local and systemic adverse-effects $[1,2]$.

Since the first use of BCG, in 1976 by Morales et al., the exact anti-tumoral mechanism has not been elucidated yet, indicating that complex immunological modulating processes involve the bacterial ablative activity on tumoral cells and an eligible and safe immunological status of the patient (ability to develop an immune response, adequate number of bacilli, close contact between BCG and tumor cells, small superficial tumor) is required to start the instillation procedure [3-7].

Authors report that BCG instillations must be avoided in patients with severe cystitis or bladder-urethral bleeding during a pre-instillation traumatic endoscopic catheterization $[6,8,9]$.

A local and systemic BCG diffusion through bladder mucosal lesions, may cause serious effects as sepsis, pulmonary and liver non caseating granulomatosis, bone marrow involvement, multiorgan failure, due to bacterial colonization and hypersensitivity reactions $[5-7,10]$.

Early antibiotic (isoniazid, rifampicin, ethambutol) tuberculostatic therapy is mandatory when a serious systemic reaction occurs within three months (early presentation) or after one year (late presentation) $[7,8,10]$.

We report a fatal sepsis and multiorgan failure occurring in a bladder carcinoma affected patient following BCG instillation procedures and discuss medico-legal implications.

\section{Case Report}

A moderate bleeding occurred in a 61 year old man, suffering from superficial bladder carcinoma, at the end of the third BCG bladder anti-tumoral instillation and after removing the endoscopic vescical instrumentation.

After 24 hours the patient accused significant urethral bleeding, malaise, nausea, arthralgias, shortness of breath, temperature (39$40^{\circ} \mathrm{C}$ ), tachycardia and shivering which partially decreased after a short antibiotic therapy (ciprofloxacin and fosfomicin during the following three days).
Seven days later the patient was admitted to hospital for acute respiratory failure with laboratory and radiological signs of lung BCG infection and sepsis, requiring isoniazid-rifampicin-ethambutol therapy.

Two days later the patient required ICU supports (mechanical ventilation, inotropic drug therapy) for BCG sepsis, ARDS, cardiovascular shock, multiorgan failure syndrome, but he died ten days later.

Post-mortem microbiological investigations on blood and serous exudates (pleural, pericardial, abdominal cavities) samples indentified the exclusive presence of bacillus Calmette-Guérin as the etiological cause of the sepsis and excluded the presence of other pathogens.

Necroscopic examination reported brain oedema, pulmonary oedema, moderate amounts of serous liquid in pleural, pericardial and abdominal cavities, abnormal liver and spleen enlargement, renal acute tubular necrosis, while diffuse miliary tuberculosis granulomas were found in lungs.

Hystological examination of granulomas showed a caseation core area surrounded by epitelioid and Langhans' giant cells.

A Polymerase Chain Reaction (PCR) analysis confirmed the presence of BCG bacterial DNA in tissue samples from caseating granulomas lesions.

A small tear with a peripherical area of chronic flogosis was identified at the posterior wall of the bladder.

The tear was far from the carcinomal focus, near the endovesical urethral meatus, while no lesions were found in the urethral wall.

*Corresponding author: Ugo Da Broi, M.D, Ph.D., Sezione Dipartimentale di Medicina Legale, Università degli Studi di Udine, Piazzale Santa Maria della Misericordia 11, 33100 Udine, Italy, Tel: 00390432 554363; Fax: 00390432 554364; E-mail: ugo.dabroi@uniud.it

Received October 20, 2012; Accepted November 26, 2012; Published November 28, 2012

Citation: Moreschi C, Da Broi U (2012) Fatal Sepsis and Multiorgan Failure Following Bacillus Calmette-Guerin Instillation for Bladder Cancer Treatment. J Forensic Res 3:171. doi:10.4172/2157-7145.1000171

Copyright: (c) 2012 Moreschi C, et al. This is an open-access article distributed under the terms of the Creative Commons Attribution License, which permits unrestricted use, distribution, and reproduction in any medium, provided the original author and source are credited. 
Forensic Authority investigated the timing and the operative proceedings of the urology surgical in order to evaluate a possible medical liability involving the death of the patient.

The bladder tear was considered as an iatrogenic lesion due to a traumatic catheterization during the third BCG instillation procedure.

The cause of death was considered as a BCG systemic diffusion through the iatrogenic lesion in the wall of the bladder, causing a fatal sepsis and multiorgan failure in absence of early diagnosis and specific antibiotic therapy.

Forensic Authority did not find written data from clinical records confirming that the patient had received exhaustive informations from the surgical team before the operation, on all possible risks and complications, normally due to the bladder bacillus Calmette-Guérin instillation, although he released the written informed consent.

\section{Discussion}

We report a case of fatal sepsis and multi-organ failure with early serious systemic adverse-effects occurring 24 hours after BCG antitumoral bladder instillation and causing diffuse systemic miliary caseating granulomas.

A delayed diagnosis and an incorrect therapeutical intervention (seven days after the BCG instillation) caused the death of the patient.

The local bacillus Calmette-Guerin immunotherapy for superficial bladder carcinoma is a clinical procedure which may produce several adverse effects and should be applied in selected patients using all the necessary cautions $[3,11]$.

The procedure should be avoided in patients with bleeding due to severe cystitis or traumatic catheterization, in order to prevent systemic BCG diffusion through bladder mucosal lesions $[3,11]$.

Minor complications (ematuria, cystitis, temperature $<38.5^{\circ} \mathrm{C}$, chills, malaise, granulomatous prostatitis, epididimo-orchitis, ureteral obstruction, bladder contracture, renal abscess) or systemic reactions (temperature $>39.5^{\circ} \mathrm{C}$, sepsis, non caseating granulomatous hepatitis and pneumonitis, arthralgias, cytopenia, bone marrow involment) are reported by Authors after BCG diffusion through bladder mucosal wall $[5,8,12-14]$.

Sepsis and non caseating granulomatous hepatitis are considered serious and uncommon events with an estimated frequency of less than $1 \%$ of all cases of BCG therapy $[5,8,12-14]$.

The early presentation of adverse-effects within three months after instillation is considered an immunocompetent host reaction with low grade pathogenic bacteria activity, while a late presentation after one year following the instillations is considered a mycobacterial reactivation [8].

Moreover Authors report that the appearance of systemic reactions with fever higher than $39.5^{\circ} \mathrm{C}$, occurring both within three months or after one year following the instillation, should be considered a life-threatening event with high mortality risk, so that all necessary diagnostic procedures to identify the BCG diffusion and the institution of antituberculous therapy (isoniazid-rifampicin-ethambutol) are priorities $[3,7,8,10,11,15]$.

In the case we report the vescical bleeding occurred at the end of the endoscopical instillation procedure, after removing the endoscopic instrumentation from the urinary anatomical ways, so that surgeons were not able to avoid or postpone the instillation and transmural diffusion of BCG in time.

The surgeons did not consider the delayed bladder bleeding, occurred at the end of the instillation procedure, as an alarm bell, suggesting the evenience of an iatrogenic mucosal lesion and the need for a strict clinical vigilance of the patient during the first days following the BCG treatment, in order to prevent local and systemic adverse effects $[3,11]$.

The behaviour of the urology surgical team was censured by Judicial Authority because of a delayed diagnosis and incorrect therapeutical intervention not applied when early local and systemic adverse effects arose after the third bladder BCG instillation.

The urology surgical team operated without cautious respect of scientific recommendations in order to prevent and treat all possible local and systemic adverse-effects due to BCG instillation and without giving the patient exhaustive pre operative informations on all risks and complications due to the procedure.

This clinical behavior was considered responsible for the death of the patient due to fatal BCG sepsis and multi-organ failure (premortem microbiological investigations on blood and serous exudates and post-mortem histological and PCR analysis from caseating granulomas lesions confirmed the presence of Bacillus CalmetteGuérin) $[3,11,16,17]$.

The early presentation of serious systemic signs and the rapid progression to a fatal sepsis and multi-organ failure, as in the case we report, have been described by Authors, but the severity of the caseating tissues lesions induced by BCG observed at microscopic examination appear really uncommon in the case we report $[1,7,8,10,11]$.

Early serious systemic adverse-effects have been reported by Authors as normally associated with non caseating granulomas in lungs and different organs and tissues [1,13,17].

In the case we report the histological examination of miliary granulomas showed a caseation core area despite optimal general conditions and normal immunological laboratory data of the patient.

The evenience of early serious systemic adverse-effects and extensive systemic miliary caseating granulomas suggests the potential occurrence of such serious istopathological lesions, so that a prudential and timely antitubercular antibiotic therapy should be mandatory in agreement with scientific recommendations $[1,13,14,16-21]$.

\section{Conclusion}

In conclusion, we observe that:

- The case we report appears an uncommon event of fatal sepsis and multiple organ failure following bacillus Calmette-Guérin instillation for bladder cancer treatment. Early serious systemic adverse-effects with diffuse miliary caseating granulomas in lungs appeared within seven days after the instillation.

- The potential evenience of early serious systemic adverseeffects and extensive systemic miliary caseating granulomas, causing a fatal clinical evolution, suggests, in agreement with scientific recommendations, that after a diagnosis of BCG systemic diffusion a timely antitubercular antibiotic therapy should be mandatory.

- Surgeons should currently give their patient exhaustive informations on all risks and complications following bladder 
Citation: Moreschi C, Da Broi U (2012) Fatal Sepsis and Multiorgan Failure Following Bacillus Calmette-Guerin Instillation for Bladder Cancer Treatment. J Forensic Res 3:171. doi:10.4172/2157-7145.1000171

Page 3 of 3

bacillus Calmette-Guérin instillation. Exhaustive information must be given the patient before the operation and release of the written informed consent.

- Claims for operative medical malpractice could be opened when BCG instillation complications occur, in absence of preoperative exhaustive informations and written informed consent, due to a delayed diagnosis and incorrect therapeutical intervention, not applied when early local and systemic adverse-effects arise.

\section{References}

1. Elmer A, Bermes U, Drath L, Büscher E, Viertel A (2004) [Sepsis and multiple organ failure after BCG-instillation for bladder cancer]. Internist (Berl) 45: 935939.

2. Mack D, Höltl W, Bassi P, Brausi M, Ferrari P, et al. (2001) The ablative effect of quarter dose bacillus Calmette-Guerin on a papillary marker lesion of the bladder. J Urol 165: 401-403.

3. Morales A, Eidinger D, Bruce AW (1976) Intracavitary Bacillus Calmette-Guerin in the treatment of superficial bladder tumors. J Urol 116: 180-183.

4. Van der Meijden AP, Sylvester RJ (2003) BCG Immunotherapy for superficial bladder cancer: an overview of the past, the present and the future. EAU Update Series 1: 80-86

5. Lamm DL, van der Meijden PM, Morales A, Brosman SA, Catalona WJ, et al. (1992) Incidence and treatment of complications of bacillus Calmette-Guerin intravesical therapy in superficial bladder cancer. J Urol 147: 596-600.

6. Rawls WH, Lamm DL, Lowe BA, Crawford ED, Sarosdy MF, et al. (1990) Fatal sepsis following intravesical bacillus Calmette-Guerin administration for bladder cancer. J Urol 144: 1328-1330.

7. Koukol SC, DeHaven JI, Riggs DR, Lamm DL (1995) Drug therapy of bacillus Calmette-Guérin sepsis. Urol Res 22: 373-376.

8. Trevenzoli M, Cattelan AM, Marino F, Sasset L, Donà S, et al. (2004) Sepsis and granulomatous hepatitis after bacillus Calmette-Guerin intravesical installation. J Infect 48: 363-364.

9. Dederke B, Riecken EO, Weinke T (1998) A case of BCG sepsis with bone marrow and liver involvement after intravesical BCG instillation. Infection 26: 54-57.

10. Lamm DL (1992) Complications of bacillus Calmette-Guérin immunotherapy. Urol Clin North Am 19: 565-572.

11. Rischmann P, Desgrandchamps F, Malavaud B, Chopin DK (2000) BCG intravesical instillations: recommendations for side-effects management. Eur Urol 37 1: 33-36.

12. Lamm DL, Stogdill VD, Stogdill BJ, Crispen RG (1986) Complications of bacillus Calmette-Guerin immunotherapy in 1,278 patients with bladder cancer. J Urol 135: 272-274.

13. Andrès E, Gaunard A, Blicklé JF, Kuhnert C, Goichot B, et al. (2001) Systemic reactions after intravesical BCG instillation for bladder cancer. QJM 94: 719.

14. Andrès E, Kuhnert C, Perrin AE, Averous G, Ruellan A, et al. (1999) [Sepsis syndrome and bone marrow granulomatosis after intravesical instillation of BCG]. Presse Med 28: 1753-1754.

15. Schnapp DS, Weiss GH, Smith AD (1996) Fever following intracavitary bacillus Calmette-Guerin therapy for upper tract transitional cell carcinoma. J Urol 156: 386-388.

16. Colombel M, Picard A (2008) Prévention des complications du Bacille de Calmette-Guérin Prevention of Bacillus Calmette-Guérin immunotherapy complications. Progres en Urologie 18: S105-S110.

17. Naoe M, Ogawa $Y$ (2008) Prevention of adverse effects of BCG immunotherapy in bladder cancer. South Med J 101: 17-18.

18. Mooren FC, Lerch MM, Ullerich H, Bürger H, Domschke W (2000) Systemic granulomatous disease after intravesical BCG instillation. BMJ 320: 219.

19. Saint F, Salomon L, Quintela R, Cicco A, Abbou CC, et al. (2002) [Classification, favorable characteristics, prevention and treatment of adverse side-effects associated with Bacillus Calmette-Guerin in the treatment of superficial bladder cancer]. Ann Urol (Paris) 36: 120-131.

20. Gonzalez OY, Musher DM, Brar I, Furgeson S, Boktour MR, et al. (2003) Spectrum of bacille Calmette-Guérin (BCG) infection after intravesical BCG immunotherapy. Clin Infect Dis 36: 140-148.

21. Lamm DL (2000) Efficacy and safety of bacille Calmette-Guérin immunotherapy in superficial bladder cancer. Clin Infect Dis 31 Suppl 3: S86-90. 